\title{
ERGODIC PROPERTIES OF AUTOMORPHISMS OF A LOCALLY COMPACT GROUP
}

\author{
M. RAJAGOPALAN
}

The following remark is made by Halmos in his book [2, p. 29]. "Can an automorphism of a locally compact but noncompact group be an ergodic measure preserving transformation? Nothing is known about this subject. Only in the compact case has anything ever been done."

The aim of this paper is to give an answer to this question of Halmos. We prove the following in this paper:

(i) A continuous automorphism $T$ of a locally compact group $G$ which is not bicontinuous cannot be ergodic.

(ii) A continuous automorphism $T$ of a locally compact group $G$ with a left invariant Haar measure $\mu$ ( $\mu$ need not be right invariant) and which is not measure preserving cannot be ergodic.

(iii) A continuous automorphism of a locally compact, noncompact abelian group is not ergodic.

Notations. We follow [2] and [3] for notions in ergodic theory and measure theory. We follow [9] and [4] for notions in topological groups. All topological spaces are assumed to be Hausdorff in this paper. Unless otherwise stated the groups occurring in this paper are not assumed to be abelian.

Definition 1. Let $G$ be a locally compact group. A continuous automorphism $T$ of $G$ is a one-to-one map from $G$ onto itself which is continuous and is also an algebraic isomorphism. An autohomeomorphism or a bicontinuous automorphism $T$ of $G$ is an algebraic isomorphism from $G$ onto itself such that both $T$ and $T^{-1}$ are continuous.

ReMARK. Some examples of locally compact groups $G$ and continuous automorphisms of $G$ which are not autohomeomorphisms are given by Robertson [8] in his thesis. To our best knowledge this is the first place where such an example is written. We give below an example of a continuous automorphism of a locally compact group which is not bicontinuous, based on an idea of K. H. Hoffman. This example shows the need of Lemma 1 below.

EXAMPLE OF A CONTINUOUS AUTOMORPHISM OF A LOCALLY COMPACT GROUP WHICH IS NOT BICONTINUOUS. Let $G_{1}$ be the circle group with the usual topology. Let $F_{1}$ be the circle group with discrete topology. Let $F_{1}=F_{2}=\cdots=F_{n}=\cdots$ and let $G_{1}=G_{2}=G_{3}=\cdots=G_{n}=\cdots$.

Received by the editors February 17, 1965. 
Let $F$ be the full direct product $\prod_{n=1}^{\infty} F_{n}$ with the usual group product and discrete topology. Let $G$ be the product $\prod_{n=1}^{\infty} G_{n}$ of the topological groups $G_{1}, G_{2}, G_{n}, \ldots$ with the product topology and usual group product. Let $H$ be the cartesian product $F \times G$ of the discrete group $F$ and compact group $G$. Then $H$ is a locally compact abelian group. We can take any element of $H$ to be a pair $(x, y)$ where $x=\left(x_{1}, x_{2}, \cdots, x_{n}, \cdots\right)$ and $y=\left(y_{1}, y_{2}, \cdots, y_{n}, \cdots\right)$ and $x_{n} \in F_{n}$ and $y_{n} \in G_{n}$ for all $n=1,2,3, \cdots$. Now define $T(x, y)=(u, v)$ where $u=\left(u_{1}, u_{2}, \cdots, u_{n}, \cdots\right)$ and $v=\left(v_{1}, v_{2}, \cdots, v_{n}, \cdots\right)$ and $v_{1}=x_{1}$ and $u_{n}=x_{n+1}$ and $v_{n+1}=y_{n}$ for all $n=1,2,3, \cdots$. Then $T$ is a continuous automorphism of $H$ which is not bicontinuous.

LeMma 1. Let $T$ be a continuous automorphism of a locally compact group $G$. Then $T$ is bicontinuous if and only if, $T^{-1}(H)$ is $\sigma$-compact whenever $H$ is an open and $\sigma$-compact subgroup of $G$ such that $H \supset T(H)$.

Proof. If $T$ is bicontinuous then $T^{-1}(H)$ is $\sigma$-compact whenever $H$ is $\sigma$-compact. Now let us assume that $T^{-1}(H)$ is $\sigma$-compact whenever $H$ is an open, $\sigma$-compact subgroup such that $T(H) \subset H$. Now let $V$ be a compact, symmetric neighbourhood of the identity of $G$. Then the group $F$ generated by $V$ is open and $\sigma$-compact. Since $T$ is continuous the sets $T(F), T^{2}(F), \cdots, T^{n}(F), \cdots$ are all $\sigma$-compact. So the group $H$ generated by $F, T(F), \cdots, T^{n}(F), \cdots$ is open and $\sigma$-compact. Clearly $T(H) \subset H$. So $T^{-1}(H)$ is $\sigma$-compact and open in $G$. By induction we get that $T^{-n}(H)$ is open and $\sigma$-compact subgroup of $G$ for all $n=1,2,3, \cdots$. So the set $S=\cup_{n=1}^{\infty} T^{-n}(H)$ is an open $\sigma$-compact subgroup of $G$ such that $T(S)=S$. So $T$ is bicontinuous on $S$ by the result of Glicksberg [1] and Pettis [5]. Since $S$ is an open subgroup of $G$ we have that $T$ is a homeomorphism of $G$.

Definition 2. Let $G$ be a locally compact group with a left Haar measure $\mu$. ( $\mu$ need not be right invariant.) Let $T$ be a one-to-one map from $G$ onto $G$. Then $T$ is called measurable if $T^{-1}(F)$ is a Borel set whenever $F$ is a Borel subset of $G$. (Here a Borel set is taken to be a member of the $\sigma$-ring generated by all closed subsets of $G$.) $T$ is said to be measure preserving if it is measurable and $\mu\left(T^{-1}(F)\right)$ $=\mu(F)$ for all Borel subsets $F \subset G$. $T$ is said to be ergodic, if it is measurable and whenever $F \subset G$ is a Borel set such that $T^{-1}(F)=F$ we have either $\mu(F)=0$ or $\mu(G-F)=0$.

REMARK. We note that a continuous automorphism of a locally compact group is measurable.

THEOREM 1. Let $G$ be a locally compact group with a left Haar measure $\mu$. Let $T$ be a continuous automorphism of $G$ which is not bicontinuous. Then $T$ is not ergodic. 
Proof. By Lemma 1, there is an open, $\sigma$-compact subgroup $H \subset G$ such that $T^{-1}(H) \supset H$ and $T^{-1}(H)$ is not $\sigma$-compact. Then $T^{-1}(H)$ should have an infinity of left cosets $\bmod H$. So there are elements $x_{0}$ and $x_{1}$ in $T^{-1}(H)$ such that $\left(x_{1} H\right) \cap H=\left(x_{0} H\right) \cap H=\left(x_{0} H\right) \cap\left(x_{1} H\right)$ $=\varnothing$. Since $H$ is $\sigma$-compact we have that $x_{0} H$ is $\sigma$-compact. Hence $T^{n}\left(x_{0} H\right)$ is $\sigma$-compact for all $n=1,2,3, \ldots$ since $T$ is continuous. So $\bigcup_{n=1}^{\infty} T^{n}\left(x_{0} H\right)$ is a Borel set where we take $T^{0}$ to be the identity. Since $x_{0} H$ is open we have that $\bigcup_{n=1}^{\infty} T^{-n}\left(x_{0} H\right)$ is open in $G$. So the set $F=\cup_{n=-\infty}^{\infty} T^{n}\left(x_{0} H\right)$ is a Borel set and clearly $T(F)=F$ and $\mu(F) \neq 0$ since $F \supset x_{0} H$ and $x_{0} H$ is open. It is easy to see from the properties of $H, x_{0} H$ and $x_{1} H$ that $\left(x_{1} H\right) \cap F=\varnothing$. So $\mu(G-F) \neq 0$. So $T$ is not ergodic.

THEOREM 2. Let $G$ be a locally compact group with a left Haar measure $\mu$. Let $T$ be a continuous automorphism of $G$ which is not measure preserving. Then $T$ is not ergodic.

Proof. By Theorem 1, we may assume that $T$ is bicontinuous. In this case both $T$ and $T^{-1}$ are measurable and there is a constant $\lambda>0$ such that $\mu(T(E))=\lambda \mu(E)$ for all Borel sets $E$, by the uniqueness of left Haar measure. Since $T$ is not measure preserving we have that $\lambda \neq 1$. So we can as well take that $\lambda>1$ (if not we consider $T^{-1}$ ). Since $T$ is not measure preserving, $G$ cannot be compact or discrete. So the measure $\mu$ is not atomic by Theorems 1 and 2 of [7]. Now let $V$ be a compact neighbourhood of the identity $e$ of $G$. Let $W=\bigcup_{n=0}^{\infty} T^{-n}(V)$. (We take $T^{0}$ to be the identity.) Then

$$
\mu(W) \leqq \sum_{n=0}^{\infty} \mu\left(T^{-n}(V)\right)=\left(\sum_{n=0}^{\infty} \lambda^{-n}\right) \mu(V)=\frac{\lambda}{\lambda-1} \mu(V)<\infty .
$$

Since $(G-W) \cap T^{-n}(V)=\varnothing$ for $n=0,1,2,3, \cdots$ we have that $V \cap\left(\cup_{n=0}^{\infty} T^{n}(G-W)\right)=\varnothing$. Now $\mu(G-W=\infty$ and $W \subset H$ where $H$ is a $\sigma$-compact open subgroup of $G$. So there is a Borel set $F \subset G-W$ such that $0<\mu(F)<((\lambda-1) / 2 \lambda) \mu(V)$. Then $\mu\left(\bigcup_{n=1}^{\infty} T^{-n}(F)\right)<\mu(V) / 2$. Hence $\mu\left(V-\bigcup_{n=-\infty}^{\infty} T^{n}(F)\right)>0$. So the set $S=\cup_{n=-\infty}^{\infty} T^{n}(F)$ is such that $0<\mu(S)$ and $0<\mu(G-S)$ and $T(S)=S$. So $T$ is not ergodic.

Lemma 2. Let $G$ be a totally disconnected, locally compact, noncompact abelian group with a Haar measure $\mu$ and $T$ a continuous automorphism of $G$ which is measure preserving. Let there exist a Borel set $S \subset G$ such that $\mu(S)>0$ and $\mu(G-S)>0$ and $S \supset T(S)$. Then $T$ is not ergodic.

Proof. By Theorem 1, we may take $T$ to be bicontinuous. Now if $G$ is discrete then the set $F=\{e\}$ has nonzero measure and $G-F$ has 
nonzero measure and $T(F)=F$, where $e$ is the identity of $G$. Hence $T$ is not ergodic in this case. So we may take that $G$ is not discrete. Now put $F=S-T(S)$. Suppose that $\mu(F)=0$. Then put $K$ $=S \cup\left(\cup_{n=-\infty}^{\infty} T^{n}(F)\right)$. Then since $\mu\left(T^{n}(F)\right)=0$ for all $n=0$, $\pm 1, \pm 2, \cdots(T$ is measure preserving) we have that $\mu(G-K)$ $=\mu(G-S)>0$. Clearly $\quad \mu(K)=\mu(S)>0$. Now $T(K)$ $=T(S) \cup\left(\cup_{n=-\infty}^{\infty} T^{n}(F)\right) \supset T(S) \cup F=S$. So $T(K)=S \cup T(K)$ $=S \cup T(S) \cup\left(\cup_{n=-\infty}^{\infty} T^{n}(F)\right)=K$ since $T(S) \subset S$. So $T$ is not ergodic in this case.

Now suppose that $\mu(F)>0$. Then we note first of all that $T^{n}(F) \cap T^{m}(F)=\varnothing$ for all $n, m=1,2,3, \cdots$ and $n \neq m$. Since $G$ is not discrete there is a Borel set $S_{1} \subset F$ such that $\mu\left(S_{1}\right)>0$ and $\mu\left(F-S_{1}\right)>0$. Now put $K=\bigcup_{n=-\infty}^{\infty} T^{n}(S)$. Then $K \cap\left(F-S_{1}\right)=\varnothing$ and $T(K)=K$. So $T$ is not ergodic in this case also.

Lemma 3. Let $G$ be a totally disconnected, locally compact, noncompact, abelian group with a Haar measure $\mu$. Let $T$ be a continuous automorphism of $G$. Then $T$ is not ergodic.

Proof. From the Theorems 1 and 2 we may take $T$ to be bicontinuous and measure preserving. Let $H \subset G$ be a compact, open subgroup of $G$. If $\mu\left(G-\bigcup_{n=1}^{\infty} T^{n}(H)\right)>0$ then we get the result by taking $S=\bigcup_{n=1}^{\infty} T^{n}(H)$ in Lemma 2 . Now suppose that $\mu\left(G-\cup_{n=1}^{\infty} T^{n}(H)\right)=0$. Now let $G_{n}$ be the group generated by $T(H), T^{2}(H), \cdots, T^{n}(H)$ where $n$ is an integer $\geqq 1$. Then $G_{1} \subset G_{2} \subset \cdots \subset G_{n} \subset \cdots$ and $\bigcup_{n=1}^{\infty} G_{n} \supset \bigcup_{n=1}^{\infty} T^{n}(H)$. So, if we put $S=\cup_{n=1}^{\infty} G_{n}$ then $S$ is an open subgroup of $G$ and $\mu(G-S)=0$. So $G=S$. So $H \subset S$. So $H \subset G_{k}$ for some integer $k$ since $H$ is compact and all the sets $G_{n}$ are open. Since $G$ is abelian we have that $G_{k}=T(H), T^{2}(H) \cdots T^{k}(H)$. So $T^{-1}\left(G_{k}\right)=H$. $T(H) \cdots T^{k-1}(H) \subset T(H) . \quad T^{2}(H) \cdots T^{k}(H), \quad T(H) \cdots T^{k-1}(H)$ $=T(H), T^{2}(H) \cdots T^{k}(H)=G_{k}$. Since $G_{k}$ is open and compact and $T$ is measure preserving we have that $T^{-1}\left(G_{k}\right)=G_{k}$. So $T$ is not ergodic again.

LEMMA 4. Let $G$ be a locally compact abelian group with a Haar measure $\mu$. Let $G_{0}$ be the connected component of the identity e of $G$ and $G / G_{0}$ be not compact. Then no continuous automorphism $T$ of $G$ is ergodic.

Proof. As in the previous lemmas, we take $T$ to be bicontinuous. Then $T\left(G_{0}\right)=G_{0}$. Now let $\phi$ be the canonical map from $G$ onto $G / G_{0}$. Let $\tilde{x}$ be a general element of $G / G_{0}$. Then define $\tilde{T}(\tilde{x})=\phi(T(x))$ where $x$ is an element of $G$ such that $\phi(x)=\tilde{x}$. Then $\tilde{T}$ is well defined on $G / G_{0}$ and is a continuous automorphism of $G / G_{0}$. So by Lemma 3 
there is a Borel set $F \subset G / G_{0}$ such that $F$ and $G / G_{0}-F$ have nonzero measure in $G / G_{0}$ and $\tilde{T}(F)=F$. Then $\mu\left(\phi^{-1}(F)\right)>0$ and $\mu\left(G-\phi^{-1}(F)\right)$ $>0$ and $T\left(\phi^{-1}(F)\right)=F$. So $T$ is not ergodic.

THEOREM 3. Let $G$ be a noncompact, locally compact, abelian group with a Haar measure $\mu$. Let $T$ be a continuous automorphism of $G$. Then $T$ is not ergodic.

Proof. Let $G_{0}$ be the connected component of the identity of $G$. Then we may as well assume that $G / G_{0}$ is compact because of Lemma 4. Then $G$ is of the form $K \times R^{n}$ where $K$ is a compact group and $n>0$. (See Theorem 41 on page 160 of [6] where this statement is proved for separable groups.) Then $T$ must be bicontinuous since $G$ is $\sigma$-compact in this case. So $T(K)=K$ since $R^{n}$ has no nontrivial compact subgroups. So we can define a continuous automorphism $\tilde{T}$ on $G / K$ such that $\tilde{T}(\phi(x))=\phi(T(x))$ for all $x \in G$ where $\phi: G \rightarrow G / K$ is the canonical map. But $G / K$ is isomorphic to $R^{n}$ and $n>0$. So by what is shown on page 28 of [2] there is a set $F \subset G / K$ of nonzero measure such that $\tilde{T}(F)=F$ and $G / K-F$ has nonzero measure. Then $\mu\left(\phi^{-1}(F)\right)$ $>0$ and $\mu\left(G-\phi^{-1}(F)\right)>0$ and $T\left(\phi^{-1}(F)\right)=\phi^{-1}(F)$. So $T$ is not ergodic.

REMARK. The Theorem 3 has been obtained by the author for all connected solvable lie groups and this result will appear elsewhere.

Acknowledgment. The author thanks Dr. W. Parry for bringing this problem of Halmos to his notice and Dr. Sankaran for some helpful conversations. This paper was done under a contract No. GP 227 from the National Science Foundation.

\section{REFERENCES}

1. I. Glicksberg, Uniform boundedness for groups, Canad. J. Math. 14 (1962), 269277.

2. P. R. Halmos, Lectures on ergodic theory, Publ. Math Soc. Japan, Kenkyusha Printing Co., Tokyo, 1956.

3. - Measure theory, Van Nostrand, New York, 1950.

4. D. Montgomery and L. Zippin, Topological transformation groups, John Wiley, New York, 1956.

5. B. J. Pettis, On continuity and openness of homomorphisms in topological groups, Ann. of Math. 52 (1950), 293-309. 1958.

6. L. S. Pontrjagin, Topological groups, Princeton Univ. Press, Princeton, N. J.,

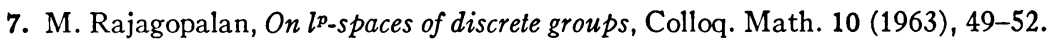

8. L. C. Robertson, Homogeneous dual pairs of locally compact abelian groups, Thesis, Univ. of California, Los Angeles, Calif., 1965.

9. André Weil, L'integration dans les groupes topologiques, Hermann, Paris, 1939.

Banaras Hindu University, India AND

UNIVERSITY OF ILLINOIS 